ÉGYPTE

monde arabe

\section{Égypte/Monde arabe}

15 | 2017

Migrations vues d'Égypte - mobilisations et politiques

\title{
Introduction - Les migrations internationales dans l'Égypte postrévolutionnaire
}

Pauline Brücker

\section{(2) OpenEdition \\ Journals}

Édition électronique

URL : https://journals.openedition.org/ema/3654

DOI : 10.4000/ema.3654

ISSN : 2090-7273

\section{Éditeur}

CEDEJ - Centre d'études et de documentation économiques juridiques et sociales

\section{Édition imprimée}

Date de publication : 5 juin 2017

Pagination : $9-26$

ISBN : 2-905838-93-0

ISSN : $1110-5097$

\section{Référence électronique}

Pauline Brücker, «Introduction - Les migrations internationales dans l'Égypte postrévolutionnaire », Égypte/Monde arabe [En ligne], 15 | 2017, mis en ligne le 05 juin 2017, consulté le 07 juillet 2022. URL : http://journals.openedition.org/ema/3654; DOI : https://doi.org/10.4000/ema.3654 


\section{Pauline Brücker}

\section{INTRODUCTION}

\section{LES MIGRATIONS INTERNATIONALES DANS L'ÉGYPTE POSTRÉVOLUTIONNAIRE}

Cix ans après l'irruption des "révolutions" arabes, des mouvements $\checkmark$ contestataires et des autres soulèvements populaires sur les scènes médiatiques et dans les débats académiques, le conflit syrien a replacé la question migratoire au cœur de l'espace méditerranéen, et questionne le rôle de l'Égypte dans cette nouvelle équation. Les départs pour l'Europe depuis la côte égyptienne, quasiment inédits avant les mouvements contestataires de 2011, sont désormais de plus en plus nombreux. Les arrivées continues depuis la Corne de I'Afrique ou la Syrie activent ces routes migratoires naissantes, empruntées par ceux et celles qui s'efforcent de contrer et de déjouer les fermetures ou la dangerosité croissante des routes anciennes, tel I'axe passant par la Libye. Les dynamiques migratoires et les stratégies de tous les acteurs en jeu - États, migrants, exilés, organisations internationales, ONG, sociétés civiles - s'adaptent ainsi à des contextes changeants et tentent de se répondre, voire de s'influencer, dans des interactions et des négociations qui restent à interroger.

En comparaison des nombreuses études dévolues à l'analyse de la scène et de la transition politiques en Égypte ou aux initiatives de la société civile, les dynamiques migratoires et leur gestion en Égypte, ont été un objet d'étude moins privilégié ${ }^{1}$. Aussi ce numéro entend-t-il problématiser la «question migratoire» en Égypte et analyser ses dynamiques anciennes et récentes. Les mouvements révolutionnaires de 2011 et en premier lieu les mouvements égyptiens ont-ils entraîné une rupture ou une continuité des pratiques au sein du champ migratoire? Plus largement, que nous révèlent les mouvements

1. L'ouvrage Égypte en révolutions (PUF, 2015) ou le dernier numéro de la revue Tiers-Monde sur l'Égypte ne comportaient aucun article sur la question migratoire. 
migratoires depuis, vers et par l'Égypte sur la situation politique et sociale actuelle du pays? Comment le pouvoir politique répond-t-il aux enjeux soulevés par des mobilités devenues conjoncturellement et structurellement plus importantes? Comment, enfin, l'État intervient-il dans un domaine qui semble en apparence peu encadré par les pouvoirs publics, ou en tout cas, qui ne représentait pas, avant la révolution de Janvier 2011, un «problème public »? Voici autant de questions auxquelles tentent de répondre cette introduction et ce numéro d'Égypte / Monde Arabe, le premier entièrement consacré aux questions de mobilité et d'exil.

\section{UN OBJET DANS L'OMBRE}

En dépit d'études sur l'émigration régionale et internationale égyptienne ou sur l'établissement en Égypte de communautés subsahariennes en provenance notamment de la Corne de l'Afrique, l'Égypte est souvent réduite à une place mineure, voire à un angle mort des recherches en sciences sociales sur les migrations internationales. Cette situation peut s'expliquer de différentes façons. Tout d'abord, l'Égypte est située entre trois zones (le Maghreb, le Machrek et le Golfe), une position géographique qui marginalise le pays par comparaison avec des systèmes migratoires régionaux plus intégrés ou plus denses, et donc traditionnellement plus couverts par les études migratoires. ${ }^{2}$ Pourtant, cet emplacement géographique particulier met justement l'Égypte au cœur de trajectoires et de parcours migratoires tout à fait singuliers, où se croisent des exilés du Machrek ou de la Corne de l'Afrique, des Égyptiens retournés d'Arabie Saoudite ou de Libye, ou encore des travailleurs bangladais ou philippins. Ces dynamiques multiformes, que I'on aurait tort de marginaliser, sont un miroir de l'Égypte du XXIe siècle et des modalités de son insertion dans des dynamiques migratoires mondialisées. ${ }^{3}$

Ensuite, si l'Égypte a toujours été une terre d'accueil pour des populations réfugiées ou déplacées suite aux grandes crises géopolitiques régionales après la création d'Israël en 1948, la guerre de Suez en 1956 ou encore les

2. Voir de Bel Air (eds), Migration et politique au Moyen-Orient, Presses de I'IFPO, 2006 ou encore H. Jaber \& F. Métral (eds), Mondes en mouvements. Migrants et migrations au tournant $d u$ XXİ̀me siècle, ouvrages collectifs où l'Égypte est quasi

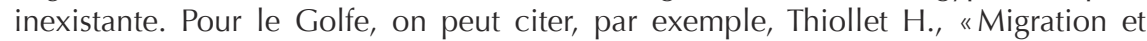
(contre)révolution dans le Golfe: politiques migratoires et politiques de l'emploi en Arabie saoudite», Revue Européenne des Migrations Internationales, 2015/3, Vol. 31, pp. 121-143.

3. Voir , Wihtol de Wenden, La Question Migratoire au XXİ̀ siècle, 2013, Presses de Sciences Po.

Castles S., de Haas H. \& Miller M., The Age of Migration, 2009, 4ème édition, Palgrave. 
guerres du Golfe de 1991 et 2003-, elle n'a jamais été le principal pays d'accueil des réfugiés du Moyen-Orient. Ainsi, des Irakiens, des Palestiniens et plus récemment des Syriens ont trouvé refuge en Égypte, mais dans aucune de ces trois situations, l'Égypte n'est apparue comme l'hôte principal, comme I'ont été le Liban, la Jordanie ou encore la Turquie dans le cas des déplacements syriens. Ceci affecte de façon substantielle la place de l'Égypte dans les mécanismes de financements régionaux et internationaux de l'asile ou des migrations. Ainsi, alors que l'Europe coopère avec nombre de pays de la rive sud de la Méditerranée depuis de longues années (Libye, Maroc, Tunisie), la place de l'Égypte au sein des dispositifs de contrôle européen est émergente (voir infra). De même, si le bureau du Haut-Commissariat aux réfugiés des Nations Unies (HCR) au Caire est également le bureau régional de cette organisation internationale, ses moyens sont bien plus limités que les antennes HCR voisines et confèrent donc à l'opération cairote une place moins stratégique. ${ }^{4}$ De fait, la représentation de l'Égypte comme un pays de destination - et pas seulement un pays de transit - est relativement récente.

Jusqu'aux années 1990, l'Égypte fût davantage traitée comme pays d'émigration et non d'immigration, ce qui était corroboré par la prépondérance des mouvements d'émigration d'alors. ${ }^{5}$ La majorité des recherches et de l'attention publique attestent de cette perspective, focalisées sur les mobilités d'Égyptiens vers les pays du Golfe, la Libye ou les États Unis, trois principaux pôles destinataires. L'importance croissante de l'émigration au sein de la société égyptienne depuis les années 1950 a fait émerger un narratif de l'exil mis en forme par différents supports artistiques, notamment le cinéma, évoqué dans les travaux

4. Néanmoins, le budget du HCR-Caire est allé croissant ces dernières années, notamment à cause de l'augmentation des demandeurs d'asiles syriens depuis 2012. En 2012, ce budget était de 25 millions. Il est passé à 63 millions en 2013, pour culminer à 85 millions en 2015. Pour 2017, le budget prévisionnel est de 79 millions. Pour plus d'informations, voir la page du HCR-Égypte, en ligne. URL : http://reporting. unhcr.org/node/2540\#_ga=1.173847843.1670485888.1478699743 (Consulté le 09/03/2017).

5. Voir Zohry, A., "The place of Egypt in the regional migration system as a receiving country." Revue Européenne des Migrations Internationales, 2003/3, Vol 19, pp. 129-149.

La nature de la question migratoire en Égypte a en effet évolué dans le temps. Zohry parle d'une première phase initiale des migrations avant 1974, au cours de laquelle l'émigration émergente dans les années 1950 devient un sujet de préoccupation politique; une seconde phase d'expansion des migrations succède de 1974 à 1984, dans un contexte économique régional marqué par le boom pétrolier et une facilitation de I'immigration égyptienne. Suivent, jusqu'au début des années 1990, une phase de contraction puis une phase de détérioration des migrations. Zohry estime que la période suivante est celle du passage à une phase d'immigration, constituée notamment par l'arrivée de dizaines de milliers de demandeurs d'asile d'Afrique subsaharienne (notamment Somalie, Soudan, Soudan du Sud, Érythrée et Éthiopie) 
de Delphine Pagès el-Karoui. ${ }^{6}$ Cette mise en avant de certaines formes de mobilité, à savoir l'émigration vers des pays riches producteurs de pétrole au détriment d'autres mobilités (les entrées croissantes de demandeurs d'asile), montre une stratégie politique de valorisation d'une certaine représentation de la migration. Certaines formes de mobilité trouvent donc crédit auprès du pouvoir politique, quand d'autres sont laissées dans l'ombre. On pense ici à la migration forcée de dizaines de milliers de Nubiens à la suite de la construction du barrage d'Assouan et de la création du lac Nasser, un épisode dont les différents aspects sont mis en lumière par Frédéric Fogel. ${ }^{7}$

Néanmoins, différentes publications témoignent d'un renouveau de I'approche de la question migratoire en Égypte. Des travaux d'anthropologie dans les années 1990 et 2000 ont mis en lumière l'importance des communautés soudanaises et subsahariennes, marginalisées et reléguées dans certains quartiers périphériques de la capitale égyptienne, comme celui d'Arba' W-Nus, dans une indifférence politique et sociale. II s'agit par exemple des études de Fabienne Le Houérou ou celles de Katarzyna Grasbka. ${ }^{8}$ La création en 2000 du Centre des Migrations et des Réfugiés ${ }^{9}$ au sein de I'Université Américaine, anciennement Programme d'étude, permet une production scientifique renouvelée sur ces questions et coïncide avec une prise de conscience graduelle de la diversité du phénomène migratoire en Égypte et de ses défis. ${ }^{10}$

6. Pagès-El Karoui D., "Les mirages de l'émigration au miroir du cinéma égyptien », Revue des mondes musulmans et de la Méditerranée, 2013, Vol 134, pp. 99-115.

7. Fogel F., Mémoires du Nil. Les Nubiens d'Égypte en migration. Paris, Karthala, 1997.

8. Voir par exemple Le Houérou F., Forced Migrants and Host Societies in Egypt and Sudan, Le Caire/New York, American University in Cairo, 2006, 112 p.; Le Houérou F. "Poussières d'instants: la reconstruction de soi et l'invention de nouveaux métiers par les réfugiées du Darfour au Caire, Mouvements, 2008/4, $n^{\circ}$ 225, pp. 81-97; Grasbka K., Marginalization in the Urban Spaces of the Global South: Urban Refugees in Cairo», Journal of Refugee Studies, 19(3), 2006, pp. 287-306; "Brothers or Poor Cousins? Rights, Policies and the Well-Being of Refugees in Egypt", in: K. Grabska \& L. Mehta (eds), Forced Displacement: Why Rights Matter, Houndsmills: Palgrave MacMillan, 2008, pp. 71-92.

9. Au départ le CMRS était le FMRS, soit le Forced Migration and Refugee Studies Program. Le programme s'est institutionnalisé et est devenu un centre à part entière au sein de I'Ecole des Affaires Publiques Internationales (School of Global Affairs and Public Policy). C'est le seul programme de Master au Caire à offrir une formation en études migratoires.

10. Les quatre axes de recherche prioritaires du centre sont assez exemplaires des arguments avancés dans cette introduction: crise actuelle des déplacements au Moyen Orient et en Afrique du Nord; question de protection (migrants et réfugiés); le futur des migrations de travail; intégration et défis posés par l'accès aux moyens de subsistance (livelihoods challenges). Pour plus d'information, voir le site du CMRS. URL: http://schools.aucegypt.edu/GAPP/cmrs/resaffl/Pages/default.aspx (Consulté le 09/03/2017). 
Différentes phases ont donc altéré un profil simpliste des mobilités en Égypte pour souligner sa place croissante comme pays d'accueil dans un système migratoire régional. ${ }^{11}$

Si la composition de ce numéro d'Égypte/Monde Arabe souligne à elle seule l'importance revêtue par la question de l'asile en Égypte, elle met également en lumière la régionalisation, sinon la mondialisation d'une crise de I'asile dont l'Égypte serait l'une des manifestations. Différents sujets sont traités dans ce numéro: la crise de l'intégration ou l'impossible naturalisation des étrangers, les politiques d'asile ambivalentes, voire contradictoires (cf. Norman), la politisation des attributions de statuts et les mobilisations sociales qui s'en suivent (cf. Brücker), les relations difficiles entre populations réfugiées et populations hôtes (cf. Mercier) ou encore les initiatives de la société civile comme palliatif aux apories de l'État (cf. Youssef). Ces thèmes ne sont-ils pas les symptômes visibles d'une crise de l'accueil et de l'asile en Égypte, mais également au sein de la rive nord de la Méditerranée?

\section{RÉVOLUTIONS ET MOBILITÉS}

Les «révolutions» de 2011 ont interpellé chercheurs et praticiens quant aux impacts sur les mouvements migratoires et les politiques de gestion des migrations. De fait, les mouvements migratoires postrévolutionnaires - de différentes natures - ont modifié les dynamiques de mobilité dans la région. Ce changement s'explique par une montée des difficultés dans les nombreux pays touchés par les mouvements contestataires de 2011, mais également par les possibilités issues d'une double déstabilisation des dispositifs de contrôle des mobilités: déstabilisation du régime politique des pays d'origine et déstabilisation des pays d'accueil, qu'ils soient situés au nord ou au sud de la Méditerranée. Cette mobilité alors rendue possible est en cela un test pour I'efficacité des dispositifs de contrôle migratoire européens et sud-méditerranéens. ${ }^{12}$ Certains chercheurs ont pu regretter que la "crise migratoire» ait surtout mis en exergue la «fonction perturbatrice» de la migration au détriment d'une analyse fine des liens structurels existants entre révolution et migration. ${ }^{13}$

11. Zohry, 2003, op cit.

12. Wihtol de Wenden C., "Migrations en Méditerranée, une nouvelle donne", Confluences Méditerranée, 2013/4, Vol. 87, pp. 19-30.

13. Schmoll C., Thiollet H. \& Wihtol de Wenden C., "Migrations en Méditerranée. Permanences et mutations à l'heure des révolutions et des crises», Migrations en Méditerranée, 2015, CNRS Edition, p. 22. 
Pour Delphine Pagès el-Karoui, plus que les révolutions, ce sont les conflits postérieurs aux mouvements contestataires qui ont généré des mouvements importants. ${ }^{14}$ Parce qu'ils sont pluriels, ceux-ci requièrent une typologie fine. En premier lieu, des départs depuis la Tunisie ou l'Égypte sont organisés dès I'année 2011 et se sont poursuivis depuis. ${ }^{15}$ Ceux-ci sont le plus souvent le fait d'hommes jeunes, avec un nombre croissant de mineurs à leurs côtés, fait inédit par son ampleur. L'Égypte est en effet le pays d'origine pour une majorité de ces mineurs. Une étude réalisée par l'OIM recense qu'en 2014, la moitié des 4095 Égyptiens arrivés illégalement en Italie étaient des mineurs non accompagnés. Ces derniers, interviewés en Grèce, cherchaient pour la plupart à se rendre en Italie (30\%), mais également en Allemagne (22\%) et en France $(17 \%) .{ }^{16}$ La fermeture des systèmes migratoires européens, dès 2011, a également généré d'importants retours d'Égyptiens (cf. Carte 1).

En deuxième lieu, les conflits en Syrie, en Libye ou encore au Yémen ont engendré des mouvements migratoires plus nombreux, qualitativement distincts puisque constitués d'hommes, mais également de familles, de femmes et d'enfants. En dépit des arrivées nombreuses en Europe à partir de 2014 et 2015, la plupart des mouvements demeurent régionaux. Ainsi, les Syriens sont aujourd'hui environ 2 millions à résider en Turquie, un million au Liban et à peu près autant en Jordanie. Les Libyens qui auraient fui la guerre et les conflits sont difficiles à compter car les mouvements sont plus diffus et souvent internes au pays. En 2015, le HCR estimait qu'il y avait environ 400000 déplacés internes en Libye, sur une population totale de 6 millions (estimation pour 2013), soit près de $8 \%{ }^{17}$

En troisième lieu, les pays traversés par des conflits suite aux contestations de 2011 ont connu des mouvements de la part des populations migrantes et réfugiées déjà présentes sur le territoire. Ainsi en va-t-il de la situation des

14. Pages el-Karoui D., "Le 'printemps arabe' : une révolution pour l'émigration égyptienne?», Revue Européenne des Migrations Internationales, 2015/3, Vol. 31, pp. 145-169.

15. Néanmoins, il faut noter ici une différence importante entre les deux pays: si des départs d'Égyptiens ont été observés dès 2011, peu sont partis d'Égypte, à l'inverse des Tunisiens. L'une des explications à cette différence est qu'en Tunisie, les dispositifs pour quitter les côtes tunisiennes vers l'Europe avec l'aide de passeurs était déjà bien rôdée au moment des révolutions, alors que la route depuis l'Égypte émerge plutôt autour de 2013. Voir sur l'exemple tunisien, Boubakri, H., «Migration et asile en Tunisie depuis 2011 : vers de nouvelles figures migratoires?», Revue Européenne des Migrations Internationales, 2015/3, Vol. 31, pp. 17-39.

16. OIM, Egyptian Unaccompagnied Migrant Children. A case study on irregular migration, Rapport en ligne, 2016.

17. Voir HCR, 2015, "Libye: le renouveau des combats provoque de nouveaux déplacements de populations», Point Presse, 16 janvier 2015. URL: http://www. unhcr.org/fr/news/briefing/2015/1/54b935dbc/libye-renouveau-combats-provoquenouveaux-deplacements-populations.html (Consulté le 09/03/2017) 

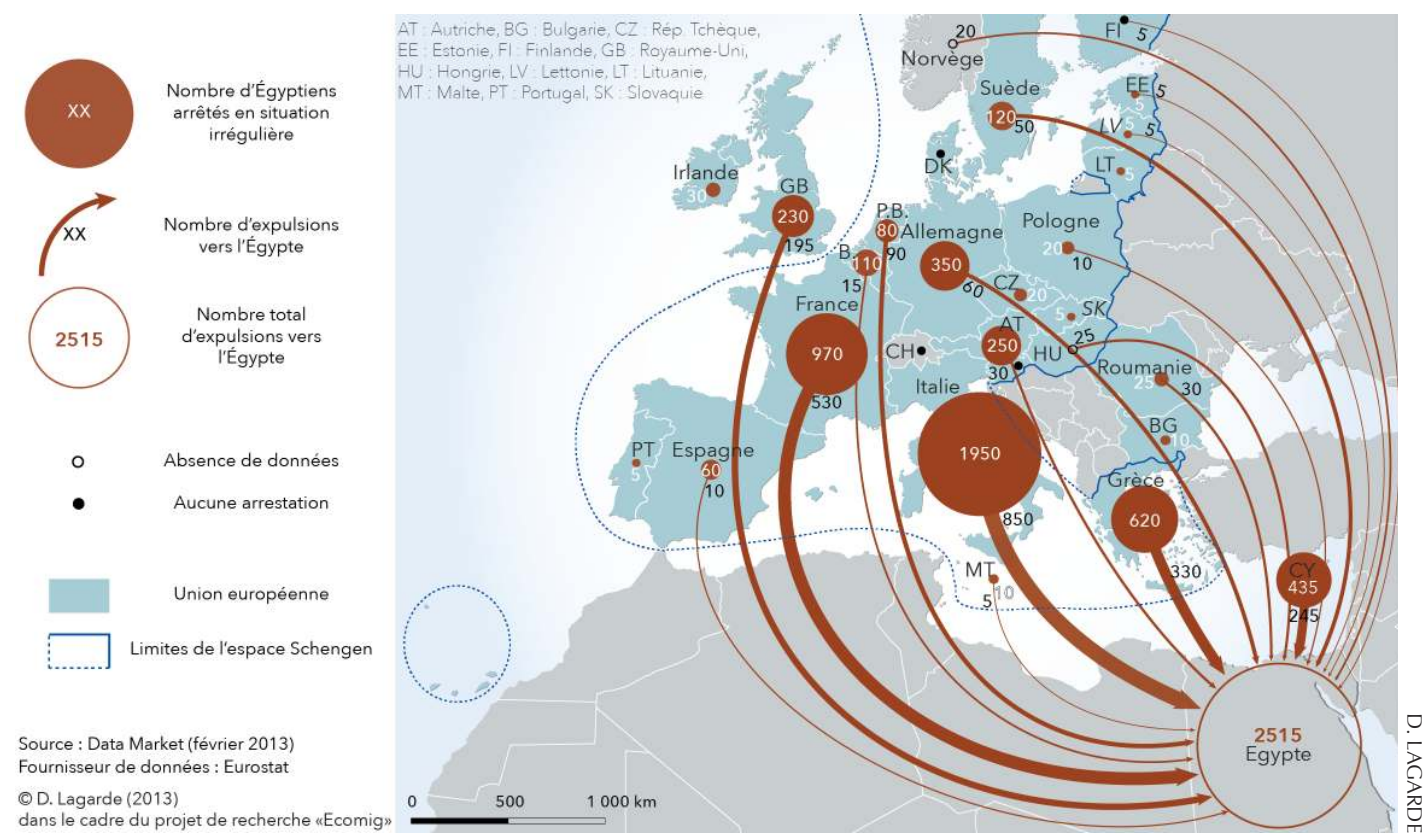

Carte 1. Expulsions d'Égyptiens hors d'Europe, en 2011

Palestiniens réfugiés en Syrie, des migrants subsahariens ou encore bangladais en Libye. Les camps temporaires de Choucha et de Saloum, installés au milieu du désert respectivement à la frontière tuniso-libyenne (2011-2013) et égypto-libyenne (2011-2014) en sont une illustration. ${ }^{18}$ De nombreux Égyptiens résidant en Libye se retrouvent contraints à un retour en Égypte. ${ }^{19}$ Ces mobilités successives se télescopent et achèvent de brouiller la frontière déjà poreuse entre les différents statuts de migrants qui définissent diverses catégories d'action publique (réfugié, travailleur migrant, retourné etc.)

En Égypte, le nombre de "personnes relevant de la compétence du HCR » passe ainsi d'environ 40000 en 2008 à 253000 en $2013^{20}$, un nombre largement inférieur aux statistiques informelles évoquées par les associations issues de la société civile et des migrants eux-mêmes. Ce décalage s'explique parce qu'un nombre important de demandeurs d'asile ne demandent pas ou n'arrivent pas à demander l'asile, pour différentes raisons: peur des représailles

18. Voir à ce titre Aguillon M-D., Lagarde, D., "Saloum, du poste frontière au camp de réfugié: les exilés de Libye deux ans après», Rapport de recherche, La Cimade, 2013. En ligne.

19. Zohry, A., "Egypt's International Migration after the Revolution: Is There Any Change?", Confluences Méditerranée, 2013/4, n. 87, pp. 47-54.

20. Voir chiffres fournis par le HCR sur la page de l'Égypte, en ligne. URL: http:// reporting.unhcr.org/node/2540\#_ga=1.173847843.1670485888.1478699743 (Consulté le 09/03/17). 
en cas de retour dans le pays d'origine, peur de se voir confisquer son passeport lors de l'obtention d'un statut de réfugié par le HCR, ou encore refus de se voir attribuer un statut humanitaire.

Une autre façon d'interroger le lien entre migration et révolution passe par la compréhension des liens entre engagement politique révolutionnaire et politisation à distance. ${ }^{21}$ La question du vote des Égyptiens dits de l'étranger (environ dix millions) a été centrale, notamment lors des scrutins de 2011 et 2012. A cet égard, Lamblin souligne que «si les émigrés égyptiens sont perçus comme une manne financière par l'État égyptien, leur implication dans la révolution égyptienne de 2011 révèle qu'ils peuvent également être une force politique aux répertoires d'actions multiples ». ${ }^{22}$ Lea Muller-Funk revient dans ce numéro sur l'évolution des politiques égyptiennes d'émigration, en montrant comment le soutien à cette mobilité va de pair avec une prise de conscience de sa dimension stratégique, à la fois politique et économique.

Une autre approche possible des migrations consiste à se placer du côté des réponses normatives apportées aux nouvelles formes de mobilité. La précarisation croissante des statuts de migrants dans le monde arabe a ainsi été mise en avant et soulignée comme un enjeu régional. ${ }^{23} \mathrm{~A}$ ce titre, certains voient dans les événements géopolitiques régionaux récents I'occasion d'une refonte nécessaire des systèmes migratoires régionaux. ${ }^{24} \mathrm{D}^{\prime}$ autres soulignent que la situation révolutionnaire égyptienne aurait peu influencé les dispositifs juridiques et institutionnels, pointant les inerties d'un système politique difficile à réformer. ${ }^{25} \mathrm{La}$ sécuritisation croissante des questions migratoires en Égypte dont nous faisons I'hypothèse (voir infra) remet néanmoins en question cette affirmation.

\section{LE RÉINVESTISSEMENT DU CHAMP DE LA MIGRATION PAR LE POLITIQUE}

Les mobilités postrévolutionnaires ont été au départ très bien accueillies dans les opinions publiques égyptiennes ou tunisiennes. L'arrivée de migrants libyens en Tunisie ou syriens en Égypte est en effet largement

\footnotetext{
21. Voir par exemple «Diasporas, migrants et exilés : quels rôles dans les révolutions et les transitions politiques du monde arabe?", colloque sous la direction scientifique de Claire Beaugrand et Vincent Geisser, organisé dans le cadre du projet de recherche WAFAW, les 16 et 17 octobre 2014 à l'IRMC, Tunis.

22. Lamblin, C., «Participation politique des Égyptiens résidant en France: vers la construction d'une citoyenneté en migration?», Revue Européenne des Migrations Internationales, 2015/3, Vol. 31, pp. 171-185, citation p. 171.

23. Pagès el-Karoui \& Boubakri, Editorial, Revue Européenne des Migrations Internationales, 2015/3, Vol. 31, pp. 17-39.
}

24. Zohry, A., 2013, op cit.

25. Pagès el-Karoui, 2015, op cit. 
soutenue. ${ }^{26}$ L'accueil semble alors marqué par une solidarité révolutionnaire. Des structures de soutien à la cause des réfugiés se créent au Caire mais surtout à Alexandrie, à l'instar du Mouvement de Soutien aux Réfugiés (Harakat el-tadamon ma'a el-lagiin) à l'initiative de la militante des droits de I'homme Mahienour el-Masri. D'autres ONG égyptiennes pour les droits de I'homme comme I'Egyptian Initiative for Personal Rights (El mobaderat el-masryeh lil hoqoq el-shakhseyeh) font progressivement place aux «migrations» dans leurs actions et leurs rapports. On peut donc - à titre préliminaire car il faudrait étayer le propos par des analyses plus conséquentes - évoquer un réinvestissement de la question migratoire par des acteurs de la société civile. Si cet engagement est à son origine intrinsèquement lié à la question syrienne, certains mouvements s'adaptent aux nouveaux enjeux, comme les départs par la mer et les nombreuses arrestations qui s'en suivent. Enfin, certaines structures moins formelles tentent de répondre aux phénomènes de marginalisation, voire de discrimination des migrants. Dans son article au sein de ce numéro, Ophélie Mercier montre d'une part les tensions qui existent dans un quartier d'Agamy où vivent Égyptiens et Syriens, et d'autre part, la façon dont l'initiative d'un groupe de spectacles de rue, Outa Hamra, cherche à faire dialoguer, par l'entremise du théâtre, ces différentes communautés.

De son côté, l'État égyptien maintient une position ambiguë, «ambivalente» à dessein pour reprendre la formule développée par Kelsey Norman dans ce numéro. La fin de la présidence de Mohamed Morsi, connue pour avoir facilité l'accueil des réfugiés syriens, s'oppose à la présidence d'Abdel Fattah al-Sissi. En effet, l'année 2013 en Égypte annonce un virage sécuritaire de la gestion des mobilités. L'éviction forcée du Président Mohamed Morsi modifie assez radicalement la situation pour les Syriens qui cherchent à rejoindre l'Égypte et pour ceux qui y résident déjà. Pour les premiers, les politiques de libre-circulation qui permettaient d'entrer sur le sol égyptien sans condition de visa sont abrogées. Pour les seconds, les suspicions de liens entre Syriens et Frères musulmans portent un coup dur à la politique spontanée de solidarité révolutionnaire précédemment évoquée. Est-ce là le jeu du nouveau gouvernement transitoire qui, au lendemain du coup d'État qui a destitué Morsi, cherche à limiter la contagion révolutionnaire, comme on a pu le voir dans le cas saoudien ${ }^{27}$ ? Maaï Youssef constate dans ce numéro que durant l'ère Morsi s'opère une politique de « décharge » des questions sociales auprès des acteurs non gouvernementaux, à travers une forme d'abandon des

26. Voir par exemple Boubakri, H., "Les migrations en Tunisie après la révolution », Confluences Méditerranée, 2013/4, n.87, pp. 31-46.

27. Sur ce point, voir Thiollet, 2016, op cit. 
dispositifs d'aide à la société civile syrienne. ${ }^{28}$ Cette politique de décharge des questions sociales serait ainsi un mécanisme structurel de l'État égyptien, un mode de gouvernance permanent au-delà des mouvements révolutionnaires.

A partir de 2013, les difficultés pour les migrants déjà présents sur le territoire égyptien s'accentuent et touchent à l'obtention de papiers de résidence que beaucoup choisissent de ne plus refaire, ou à un sentiment persistant d'insécurité. Le traitement différencié entre différentes nationalités (Syriens vs. Subsahariens notamment) alimentent des conflits entre migrants. Les conditions de vie précaires mêlent difficultés financières, difficulté à l'embauche, racisme institutionnel, discriminations quotidiennes, difficulté d'accès à l'éducation pour les enfants et les adultes ainsi que difficulté au logement. Cette situation pousse un nombre croissant de gens sur les dangereuses routes de l'Europe (voir infra) ou d'Israël.

C'est paradoxalement cette dangerosité qui justifie du côté de l'État une politique sécuritaire des migrations. D'un côté s'institutionnalise à partir de 2005 une nouvelle route vers l'est: celle qui depuis le Caire et l'est soudanais, rejoint le désert du Sinaï et celui du Néguev en Israël (carte 2). Cette route amène Israël et l'Égypte à coopérer dans un objectif d'entraver ces mouvements par la force. Ainsi, l'Égypte met rapidement en place une politique dite de «tir pour arrêter» («shoot-to-stop», en anglais), donnant lieu à des blessures par balles sur les migrants mais également des arrestations, des procès expédiés devant des cours militaires et des déportations, pourtant illégales en droit international. ${ }^{29}$ En parallèle, des trafics d'êtres humains sans équivalent dans l'histoire du pays donnent naissance à des maisons de torture dans le Sinaï, où stationnent dans des conditions effroyables des dizaines de candidats au départ pour Israël. ${ }^{30}$

Des naufrages à répétition se succèdent depuis les routes de l'est méditerranéen, notamment à partir de 2013. En 2015, on estime qu'environ un million de personnes auraient accosté en Europe: 5350 individus auraient perdu la vie lors de la traversée. Plusieurs naufrages ont eu lieu depuis ou près de l'Égypte. En septembre 2016, dans un des plus graves accidents survenus depuis l'Égypte, 200 personnes ont trouvé la mort au large de Rosetta après le

28. Voir sur ce point, Ben Nefissa, S., "Les dynamiques sociales et politiques paradoxales de la promotion de la société civile en Égypte», in Anna Bozzo et al., Les sociétés civiles dans le monde musulman, La Découverte, 2011, p. 325-340 ou encore Ben Nefissa, Sarah, "Les dynamiques sociales et politiques paradoxales de la promotion de la société civile en Égypte», in Anna Bozzo et al., Les sociétés civiles dans le monde musulman, La Découverte, 2011, p. 325-340.

29. Human Rights Watch, "Sinai Peril. Risks to migrants, refugees and asylum-seekers in Egypt and Israel", Novembre 2008.

30. Voir par exemple Human Rights Watch, "I wanted to lie down and die'. Trafficking and torture of Eritreans in Sudan and Egypt", Février 2014. 


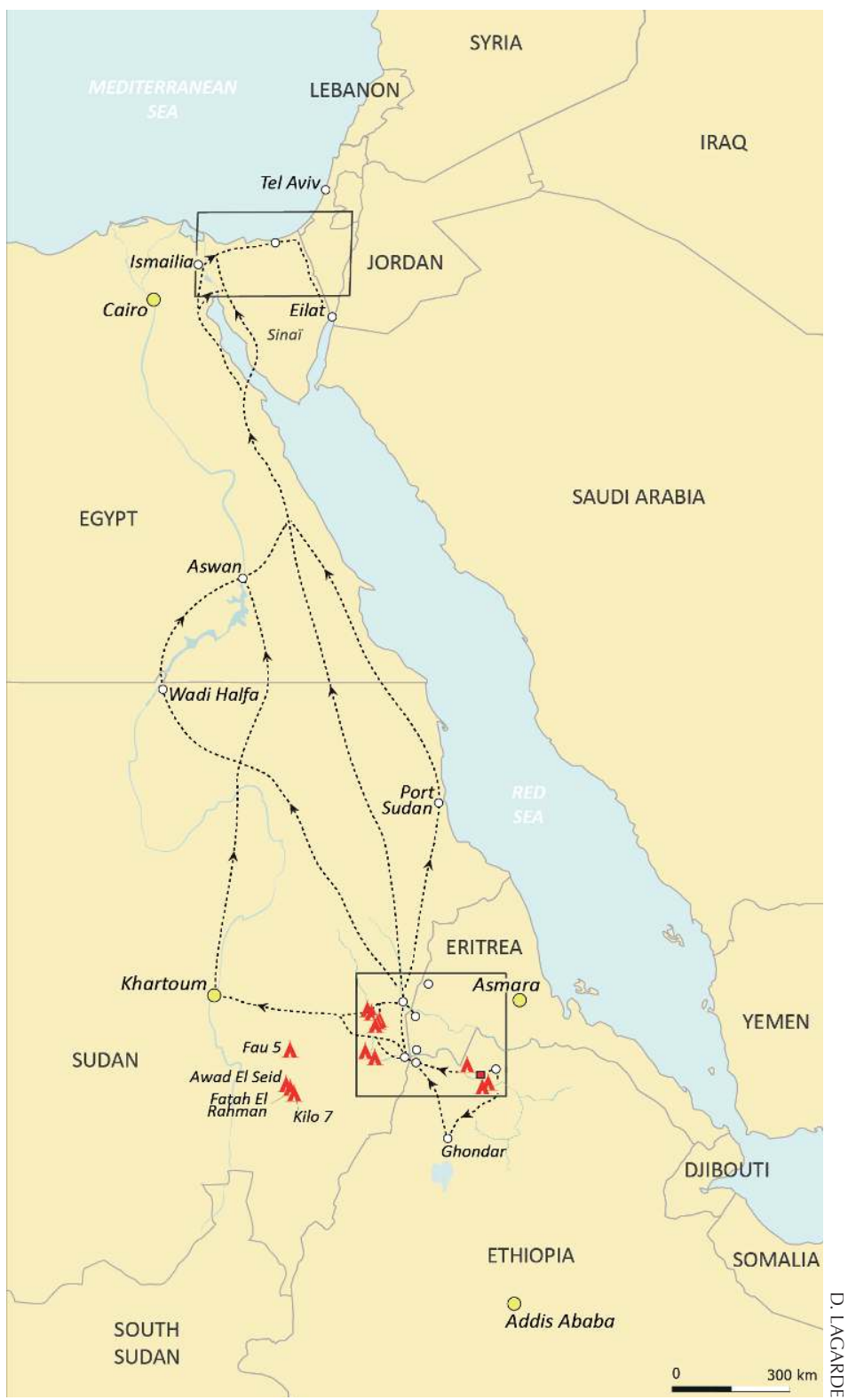

Carte 2. Les routes migratoires principales depuis l'érythrée jusqu'au Sinaï. Voir page suivante pour les encadrés. 

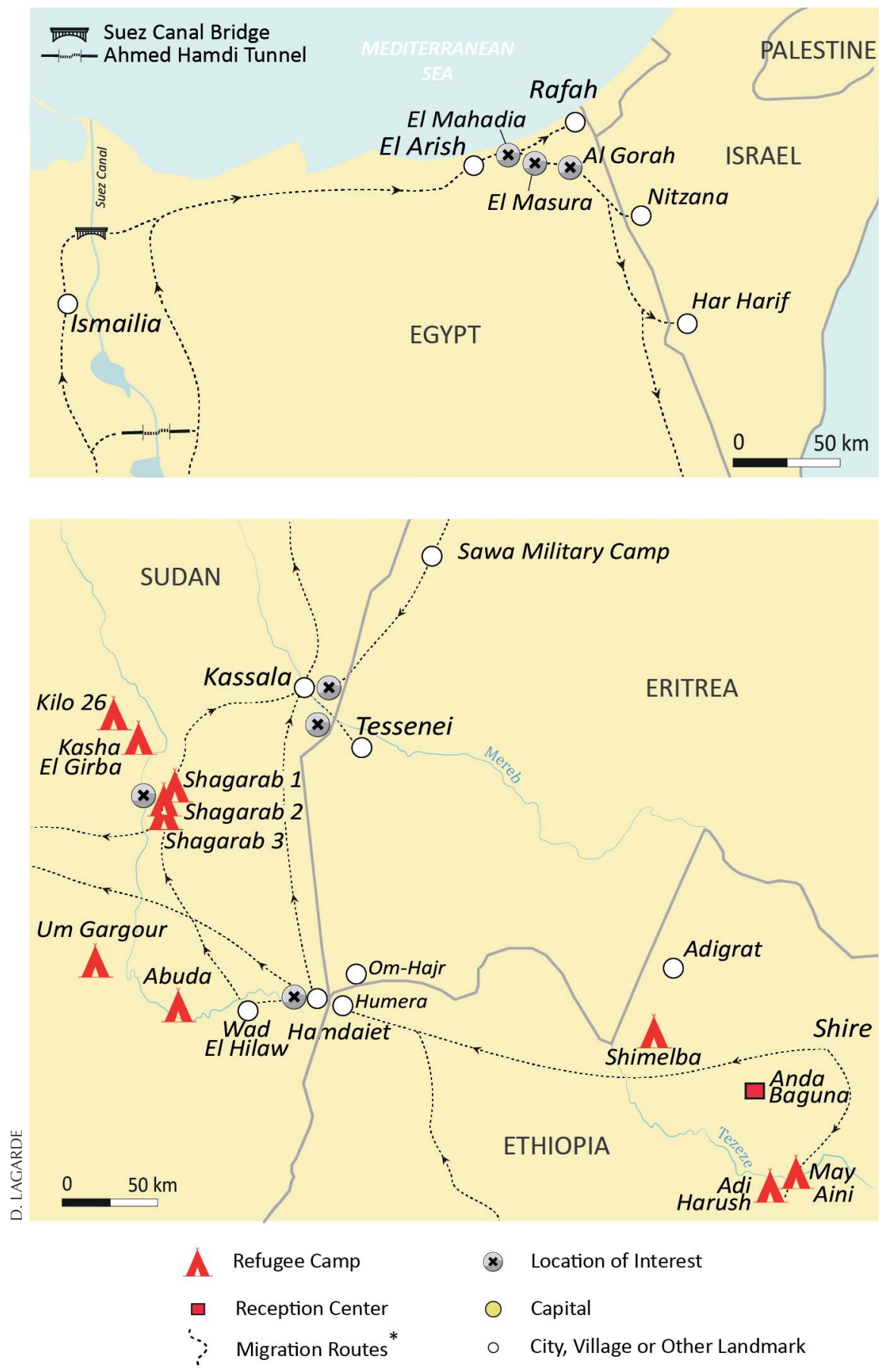

* These maps represent approximations of the

Carte 2. Les routes migratoires principales depuis l'Érythrée jusqu'au Sinaï. 
naufrage d'un bateau qui transportait près de 450 personnes. ${ }^{31}$ Certains demandent «où se trouvait l'État?» lors de l'accident, soulignant une politique à deux vitesses en matière de migration, hâtive à sanctionner mais lente à secourir. ${ }^{32}$

Ces deux phénomènes, ces deux routes et leurs lourds bilans, ont entraîné l'État égyptien vers une refonte de son discours de la migration et vers un réinvestissement sécuritaire. De nouvelles institutions ont été créées, à l'instar du Comité national interministériel égyptien de coordination pour combattre et prévenir la migration illégale (NCCPIM) ${ }^{33}$ établi par Décret ministériel en 2014 afin de coordonner les efforts nationaux de lutte contre la migration irrégulière depuis, par et vers l'Égypte. Ce comité interministériel est directement lié à l'OIM qui a soutenu l'initiative depuis sa création. ${ }^{34}$

Ce comité a adopté un plan d'action pour 2014/2015 ${ }^{35}$, puis en 2017 un plan d'action stratégique sur dix ans, intitulé «Prévenir et combattre la migration irrégulière en Égypte (PRIME en anglais) » ${ }^{36}$, soutenu par le Bureau britannique des affaires étrangères et du Commonwealth, en coopération

31. Voir HCR, "Tragédie en mer pour des réfugiés au large de l'Égypte», Communiqué de presse, 23 septembre 2016, En ligne. URL: http://www.unhcr.ch/fr/presse/artikel/8 f5b0ce918292399704a4ead905aeae3/tragedie-en-mer-pour-des-refugies-au-largede-lÉgypte.html (Consulté le 07/03/2017).

32. Voir Magid P. "Report from Rashid: Where was the state when hundreds drowned?", Mada Masr, 13 octobre 2016. En ligne. URL:

http://www.madamasr.com/en/2016/10/13/feature/politics/report-from-rashidwhere-was-the-state-when-hundreds-drowned/ (Consulté le 10/03/2017).

33. "The National Coordinating Committee on preventing and combating illegal migration (NCCPIM) is considered the focal point for policies and governmental efforts in the field of combating and preventing illegal migration, ensuring the coordination among governmental entities represented in it and the other nongovernmental actors, it also acts as an advisory body to the national agencies and authorities." Voir le texte de constitution de Comité, Site du Ministère des Affaires Etrangères, en ligne, URL:

http://www.mfa.gov.eg/English/Ministry/TraffickinginPersons/committee/Pages/ NCCPIM.aspx (Consulté le 07/03/2017).

34. OIM, «L'Égypte intensifie ses efforts pour lutter contre la migration irrégulière», 15 janvier 2016, En ligne. URL:

http://www.iom.int/fr/news/lÉgypte-intensifie-ses-efforts-pour-lutter-conter-lamigration-irreguliere (Consulté le 07/03/2017).

35. NCCPIM, "Action plan 2014-2015», En ligne. URL: http://www.mfa.gov.eg/ Arabic/InsideEgypt/economyindicator/Documents/NCCPIM\%20Action\%20Plan\%20 2014-2015.pdf (Consulté le 10/03/2017).

36. National Coordinating Committee on Combating and Preventing Illegal Migration (NCCPIM), "National Strategy for Combating Illegal Migration 2016-2026", En ligne. URL: http://www.awaremigrants.org/wp-content/uploads/2017/01/National-Strategyfor-Combating-Illegal-Migration-EN.pdf (Consulté en ligne 09/03/2017). 


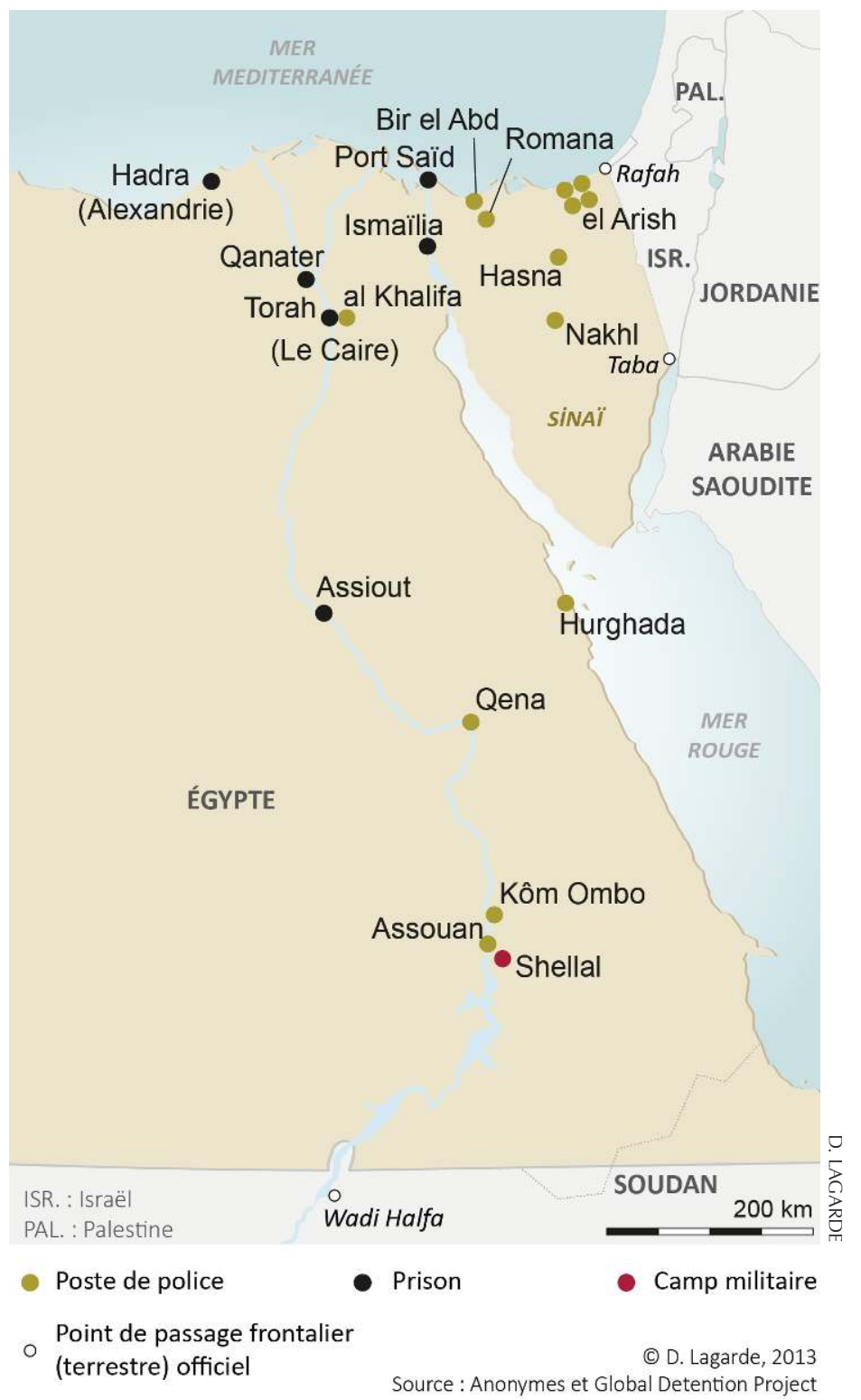

Carte 3. Principaux lieux d'enfermement d'étrangers en Égypte.

En pratique, chaque prison ou poste de police en Égypte peut-être utilisé de manière non-officielle pour l'enfermement administratif des étrangers. Seules les prisons de Torah, el Qanater, Hadra et Port Saïd, ainsi que le poste de transit d'al Khalifa sont enregistrés dans la loi comme lieux de rétention administrative pour les étrangers en attente d'expulsion (décret 659 de 1986). Cette carte ne se veut donc pas exhaustive, mais cherche à montrer les principaux lieux utilisés pour l'enfermement des étrangers en Égypte au cours de l'année 2012. 
avec l'OIM. ${ }^{37}$ Si le plan prévoit des activités de sensibilisation ${ }^{38}$ ainsi que des aides financières, il a pour objectif premier la lutte contre les passeurs et les départs illégaux. Ce comité a été également à I'initiative d'un projet de loi adopté en octobre 2016, qui vise à réprimer les passeurs afin de réduire l'immigration illégale par l'Égypte. Le projet de loi entend ainsi punir les passeurs d'une peine de prison ainsi que d'une amende allant de 200000 à 500000 livres égyptiennes. ${ }^{39}$ Si le terme de «droit de l'homme» ponctue les textes et documents constitutifs, la façon dont ces mesures seront mises en place inquiète au vu de la répression des départs illégaux menée par les forces de police égyptiennes ces dernières années. ${ }^{40}$

Ainsi, loin d'une refonte de la compréhension de la question migratoire, on semble bien dans un scénario de reprise des «procédés classiques de 'lutte contre l'immigration clandestine' ${ }^{41}$. Le cœur des pratiques est en effet toujours la répression des mouvements illégaux, à l'instar des mesures d'enfermement des migrants ayant tenté de partir par la mer, dans la région

37. Voir OIM, «L'Égypte lance un nouveau projet de lutte contre le trafic illicite de migrants », Communiqué de presse, 14 Février 2017. En ligne. URL: http://www.iom. $\mathrm{int} / \mathrm{fr} /$ news/legypte-lance-un-nouveau-projet-de-lutte-contre-le-trafic-illicite-de-migrants (Consulté le 09/03/2017).

38. Le coût estimé de cette campagne menée dans onze gouvernorats égyptiens est chiffré à environ 10 millions de livres égyptiennes.

39. La loi se compose de 34 articles. Les articles 5, 6, 7, 8 et 12 imposent des peines d'emprisonnement et une amende maximale de 500000 LE envers les personnes reconnues coupables de trafic de migrants potentiels; agissant comme courtiers ou intermédiaires pour les contrebandiers; ou fournissant un abri aux migrants illégaux ou victimes de la traite; Ou rassemblant, transportant ou facilitant la traite des migrants. L'article 22 encourage la coopération judiciaire entre l'Égypte et d'autres pays. L'article 25 vise à protéger les migrants, en particulier les femmes et les enfants, en leur fournissant un traitement humanitaire et l'accès aux soins de santé et à l'assistance juridique. L'article 28 établit un comité chargé de coordonner les différents efforts nationaux et internationaux de lutte contre la migration illégale et la traite des êtres humains.

40. Une fillette de huit ans a été par exemple tuée en août 2015, alors qu'elle était sur un bateau prenant le large, près de Borg al-Burullus, dans le gouvernorat de Kafr el-Sheikh. Pour plus d'informations, voir Smith, "Egypt's crack down on irregular migrants », Middle East Eye, 21 août 2015. En ligne. URL: http://www.middleeasteye. net/news/syrian-minor-shot-dead-egypt-s-coast-government-eyes-greater-bordersecurity-41741478 (Consulté le 09/03/2017).

41. Pages el-Karoui, D. \& Boubakri, H., 2016, op cit. 
$\mathrm{d}^{\prime}$ Alexandrie ${ }^{42}$ (carte 3 ). Ce contexte confirme une utilisation stratégique de la question migratoire où «le prétendu 'problème' de l'immigration fournit la variable d'ajustement des politiques générales " ${ }^{43}$ : le contexte révolutionnaire aurait finalement servi la cause d'une politique publique restrictive et autoritaire. On dépasse ainsi la dichotomie investissement/désinvestissement de l'État par ce qui serait précisément un jeu stratégique et permanent entre investissement et désinvestissement, ce que Kelsey Norman appelle «l'ambivalence» de la politique égyptienne (voir Norman, dans ce numéro). Enfin, les questions statutaires permettent également de souligner une certaine prégnance dans la politisation des modes de gestion des migrations: différenciation des prises en charge en fonction des nationalités et préférence portée à l'égard des populations arabes, principalement syrienne et libyenne. ${ }^{44}$

\section{L'ÉGYPTE, NOUVELLE TERRE D'EXTERNALISATION DU CONTRÔLE FRONTALIER EUROPÉEN}

Si l'Égypte coopère depuis plusieurs années déjà avec son voisin israélien, la coopération avec I'Union Européenne est bien plus récente. L'Égypte entend-elle devenir l'un des nouveaux pions du dispositif européen d'externalisation du contrôle des frontières? L'augmentation des départs par l'Égypte, ainsi que sa place de carrefour migratoire de plus en plus fréquenté, ont nourri les intérêts européens à la recherche d'un nouveau partenaire. La fin des politiques de contrôle instaurées avec la Libye après la chute du régime de Kadhafi, a accentué le rôle stratégique de substitution que pouvait jouer l'Égypte. A l'inverse, ce pays semble avoir bien compris l'intérêt financier que pouvait représenter ce nouveau rôle, et n'hésite pas à s'en servir quand le gouvernement rappelle avoir sur son territoire près de 5 millions de migrants, sous-entendant qu'il faudrait un rien pour que ces millions arrivent en

42. A l'instar du tragique cas de Karmooz, du nom du commissariat où une soixantaine de migrants est restée enfermée pendant près d'un an. Pour plus d'informations voir par exemple, El Gundy E., «In limbo: Refugees held for months at Alexandria police station», Ahram Online, 15 Mars 2015. En ligne. URL: http://english.ahram. org.eg/NewsContent/1/64/125357/Egypt/Politics-/In-limbo-Refugees-held-formonths-at-Alexandria-po.aspx (Consulté le 10/03/2017).

43. Brun, 2016

44. A cet égard, la décision de l'Égypte de ne pas faire reconnaitre par le HCR les ressortissants libyens comme réfugiés est exemplaire des traitements de faveur dont est capable l'État à l'égard d'une catégorie de population dont il souhaite favoriser les conditions de vie, notamment par des facilitations de type administratifs. De la même façon, la décision de ne pas faire reconnaître les Palestiniens dits de Syrie, s'inscrit dans une politique de perpétuation du «droit au retour», qui conduisit de nombreux pays de la région à ne pas faire reconnaître les Palestiniens comme réfugiés. 
Europe. ${ }^{45}$ On assiste à un marchandage propre à l'externalisation européenne, mais relativement nouveau en Égypte, où les programmes de développement s'échangent contre des projets de lutte contre l'immigration. ${ }^{46}$

Via I'OIM mais également via certains gouvernements (Italie, Royaume Uni), l'Europe tente de s'imposer comme acteur de contrôle et d'influence des politiques migratoires égyptiennes. Les différentes initiatives précédemment citées ont été largement soutenues sinon encouragées par l'Europe. L'institutionnalisation de la question de l'immigration qui devient progressivement une question publique en Égypte, est encouragée par I'UE et s'incarne dans différents projets en cours.

Un programme intitulé «Enhancing the Response to Migration Challenges in Egypt (ERMCE)» prévoit de "renforcer la gouvernance migratoire de l'Égypte» par le renforcement des capacités des agences gouvernementales et $\mathrm{d}^{\prime}$ «accroître la protection et les opportunités socio-économiques pour les migrations actuelles ou potentielles » dans la perspective de freiner les choix migratoires. ${ }^{47}$ Le programme n'a pas encore été lancé pour l'instant, faute de désaccord entre Bruxelles et le Caire quant à ses modalités pratiques, mais il permet néanmoins de prendre le pouls d'un partenariat institutionnel en expansion. De son côté, le Processus de Khartoum déborde l'Égypte pour traiter régionalement de la route est-africaine vers l'Europe avec comme partenaires le Soudan, l'Éthiopie ou encore l'Érythrée. L'objectif du programme est également de renforcer les capacités nationales dans un but de contrôle des frontières et des mouvements migratoires. Les modalités pratiques des allocations de fonds sont encore floues, même si le processus depuis 2016 gagne progressivement en institutionnalisation. ${ }^{48}$

45. Voir par exemple les propos de Naela Gabr, présidente du NCCPIM, «Fighting the Waves: Egypt's response to the global challenge of Illegal Migration», Blog du Ministère des Affaires Etrangères égyptien. En ligne. URL:

https://mfaegypt.org/2016/10/08/fighting-the-waves-egypts-response-to-the-globalchallenge-of-illegal-migration/ (Consulté le 10/03/2017).

46. Sur l'Égypte en particulier, voir S. Philip Naceur \& T. Rollins, «Europe's migration trade with Egypt», Mada Masr, ${ }^{\text {er }}$ février 2017. Sur le phénomène d'externalisation plus largement, voir par exemple El-Qadim, N., Le gouvernement asymétrique des migrations. Maroc/Union Européenne, 2015, Dalloz.

47. Ce programme dispose d'un budget d'11,5 millions d'euros. Voir le site du projet, «Enhancing the response to migration challenges in Egypt», Département de coopération Internationale et développement. URL: http://ec.europa.eu/europeaid/trustfund-projects/ermce_en (Consulté le 10/03/2017). Voir également Naceur \& Rollins, op. cit.

48. Voir par exemple le tout récent site du Processus: http://www.khartoumprocess. net/ (Consulté le 10/03/2017). 
L'Égypte, à la faveur d'une augmentation des départs par le Sinaï ou la Méditerranée, a ainsi opéré un tournant sécuritaire de sa gestion migratoire, déjà largement à l'œuvre dans le Sinaï avant les mouvements révolutionnaires de 2011. Si le système n'a donc pas radicalement changé, nous reconnaissons des orientations sécuritaires plus fortes et plus institutionnalisées que par le passé, qui devront être analysées. Ainsi, plutôt que de réorienter sa politique, l'Égypte semble avoir utilisé un contexte d'opportunité politique qui lui était favorable pour faire ainsi coïncider répression interne et répression aux frontières. La politique de restriction des migrations illégales entérine une approche sécuritaire et stratégique du fait migratoire. De fait, loin d'être absent de la gestion des migrations, l'État en délaisse stratégiquement certains pans, comme l'attribution de statut de réfugié, géré par le HCR ou bien l'aide et l'assistance, gérées en partie par des structures communautaires.

Dès lors est posé ce qui était l'une des ambitions de ce numéro: réinterroger le rôle et la place du politique au sein du champ migratoire, une considération souvent délaissée au profit d'une approche géographique ou anthropologique des migrations. Or nous l'avons dit, au-delà d'un «laissez-faire» de façade, l'Égypte joue un rôle bien plus politique dans sa gestion des mobilités que ne veut le laisser croire le discours d'État. Par ailleurs, les communautés migrantes, qu'elles soient égyptiennes à l'extérieur du pays ou étrangères sur le sol égyptien, tentent de s'affirmer comme acteur politique local, et laissent peut-être présager ici un questionnement à venir sur les systèmes d'intégration politique des populations étrangères en Égypte, aujourd'hui inexistant. 